\title{
Understanding Suicidal Behavior: The Contribution of Recent Resting-State fMRI Techniques
}

\author{
Gianluca Serafini ${ }^{*}$, Matteo Pardini ${ }^{2}$, Maurizio Pompili ${ }^{3}$, Paolo Girardi ${ }^{3}$ and Mario Amore ${ }^{1}$ \\ ' Section of Psychiatry, Department of Neuroscience, Rehabilitation, Ophthalmology, Genetics and Maternal and Child \\ Health, University of Genoa, Genoa, Italy, ${ }^{2}$ Section of Neurology, Department of Neuroscience, Rehabilitation, \\ Ophthalmology, Genetics and Maternal and Child Health, University of Genoa, Genoa, Italy, ${ }^{3}$ Department of Neurosciences, \\ Suicide Prevention Center, Sant'Andrea Hospital, University of Rome, Rome, Italy
}

Keywords: suicidal behavior, resting state, rsfMRI, functional deficits, brain networks

\section{INTRODUCTION: SUICIDAL BEHAVIOR AND RESTING-STATE fMRI TECHNIQUES}

OPEN ACCESS

Edited by:

Stefan Borgwardt,

University of Basel, Switzerland

Reviewed by:

Johannes Sebastian Wrege, Psychiatric Clinic of University of

Basel, Switzerland

Kees Van Heeringen,

Ghent University, Belgium

*Correspondence:

Gianluca Serafin

gianluca.serafini@unige.it

Specialty section:

This article was submitted to

Neuroimaging and Stimulation,

a section of the journal

Frontiers in Psychiatry

Received: 10 February 2016 Accepted: 06 April 2016

Published: 19 April 2016

Citation:

Serafini G, Pardini M, Pompili M,

Girardi $P$ and Amore M (2016)

Understanding Suicidal Behavior: The

Contribution of Recent Resting-State

fMRI Techniques.

Front. Psychiatry 7:69.

doi: 10.3389/fpsyt.2016.00069
Suicidal behavior is a relevant and multifaceted public health issue and is commonly associated with a significant disability and psychosocial impairment. To date, no available biomarkers are able to predict which subjects will develop suicide over time, and this is hardly surprising given the number of factors that have been hypothesized to modulate suicide risk based on the current literature (1).

In the effort to solve this shortcoming, a possible approach is represented by the search of those patterns of brain activation that are associated with suicidal behavior and may be identified using functional neuroimaging techniques. To date, the most commonly used functional neuroimaging technique is represented by functional magnetic resonance imaging (fMRI). fMRI may detect the local changes in the relative concentrations of oxy- and deoxy-hemoglobin, induced by local metabolic demand [i.e., it measures the so-called blood-oxygen level-dependent (BOLD) signals] (2). fMRI data can be also acquired while the imaged subject is performing a given task (i.e., taskdependent fMRI) or at rest (resting-state fMRI - rsfMRI).

There are studies showing aberrant neural activity patterns in suicide attempters that were carried out using task-based BOLD fMRI (3). Indeed, task-based fMRI has been used to probe the neural substrates of specific cognitive and emotional intermediate phenotype of suicide, such as error monitoring (4) and decision-making (5), but task-based fMRI is inherently limited by the need of active collaboration by the scanned subject as well as by the nature of the task during fMRI data acquisition. fMRI data can be also acquired while the subject is not performing any task - i.e., at rest (rsfMRI) - to evaluate which brain regions present same patterns of activation over time that are supposed to represent a valid surrogate marker of functional connectivity between different gray matter areas and over the whole brain (6).

Compared with task-based fMRI, rsfMRI is not dependent on subject collaboration (except for the requirement to lay in the scanner as much as possible), thus increasing its inter-subject and intra-subject reproducibility. Moreover, rsfMRI allows to explore the resting-state brain networks, in particular, the default mode network (DMN), that have been reported to be altered in several psychopathological conditions and may be not easily investigated using the commonly available task-based fMRI $(7,8)$. Finally, as rsfMRI data can be analyzed over the whole brain, they do not require to have an a priori hypothesis regarding the involvement of specific brain regions. 


\section{CAN rSFMRI INFORM ABOUT SUICIDAL BEHAVIOR?}

In order to perform a critical overview of the existing studies about the main topic, a reference search was carried out across the Medline and ScienceDirect databases (January 1980 and February 2016). The search used the following terms: "Resting-state fMRI" OR "Resting-state functional magnetic resonance imaging" OR "rsfMRI techniques" AND "Suicid*" (including suicidal behavior OR suicide ideation OR suicidal thoughts OR deliberate self-harm OR suicidal attempt). In addition, the reference lists of all papers identified were reviewed, and imaging evidence investigating suicidality as a secondary emergence of disturbed experience of self in personality disorders have been also included.

Although fMRI may investigate brain activity both in resting conditions and during activation, in the present paper, we mainly focused on brain imaging in resting conditions estimating regional brain activity when environmental activation is standardized. fMRI studies that investigated depressed patients pointed to the possible role of a host of different regions in this complex construct. Interestingly, all these brain areas have been also shown to play a role in different psychopathological domains such as modulation of physiological responses to emotions, emotional dysregulation, and self-processing, which in turn are also supposed to play a role in the emergence of suicide behavior $(3,5)$. As reviewed by Desmyter and colleagues (9), the reduced perfusion of the prefrontal cortex in suicidal patients is a commonly observed finding in functional neuroimaging resting conditions.

Indeed, the search for the neural bases underlying the cognitive and emotional intermediate phenotypes may reveal interesting neurocognitive constructs concerning suicidality. Cao et al. (10), for example, aimed to explore changes in neural circuit organization associated with suicidal behavior and proposed that disruptions in fronto-limbic or fronto-parietal-cerebellar pathways may lead to poor executive functioning, lack of impulse control, cognitive inflexibility, and impaired decision-making in suicidal young adults.

Very recently, Northoff (11) reported that depressive symptoms may be interpreted as spatiotemporal disturbances of the resting-state activity and its spatiotemporal structure according to the general assumption that brain resting-state activity may be defined in a functional/physiological manner rather than anatomically/structurally. Based on this suggestion, ruminations and enhanced self-focus in depressed patients are referred to abnormal spatial organization of resting-state activity, whereas anhedonia and suicidal ideation may be associated with increased focus on the past and enhanced past-focus as basic temporal disturbances of the resting state. Aiming to investigate the neural correlates of suicide thoughts, fMRI during presentation of autobiographical memories in depressed patients who recently attempted suicide has been also carried out. A deactivation of frontal cortical areas has been observed in suicidal episodes (mental pain plus suicide action), whereas an increased neural activity in the medial prefrontal, the anterior cingulate cortex, and the hippocampus occurred during the recall of the suicide action compared to mental pain (12). The authors suggested that suicidal mode is a state-dependent phenomenon that can be triggered by a specific stimulus and it may possess the quality of a traumatic state.

There are studies that indirectly addressed suicidal behavior using rsfMRI techniques [e.g., they mainly investigated relevant predictors of suicidal behavior such as hopelessness $(13,14)]$, and provided useful information about suicidality. Hopelessness is a powerful and informative psychological construct about suicidal behavior and addressed three major constructs: feelings regarding the future, loss of motivation, and expectations (15).

Interestingly, Northoff and colleagues (16) suggested that hopelessness and self-related processing were associated with higher resting-state neural activity in the DMN. Grimm and colleagues (17) aimed to investigate whether self-related emotional judgment may induce reduced negative BOLD responses (NBRs) in DMN regions of depressed patients. Reduced NBRs in the anterior regions of the DMN associated with abnormally increased self-focus together with ruminations, self-blame, abnormal coupling of the self to negative emotions, and enhanced attention to the own self were found among depressed patients relative to healthy individuals (18). Interestingly, higher self-relatedness of negative stimuli was also associated with hopelessness measured using the Beck Hopelessness Scale. This is in line with the observation that, clinically depressed patients usually present alterations in anticipating the future, but they also show increased retrieving and ruminating about past events (19). The study of Grimm and colleagues (17) postulated the existence of a dysregulated balance between anterior and posterior medial regions of NBRs during self-related judgment that may suggest a shift in the functional balance between anticipation and retrieval, or future and past.

Figure 1 showed the potential of rsfMRI techniques to inform about vulnerability to suicidal behavior. We mainly focused on self-relatedness of negative stimuli that, according to Grimm and colleagues (17), are predominantly related to reduced NBRs in the anterior regions of the DMN and increased vulnerability to suicidal behavior, whereas we omitted to mention the role of motivational and consummatory anhedonia that are presumably associated with reward network dysfunctions [for more details about this topic, see the recent review of Lener and Iosifescu (20)].

Further additional details need to be discussed in this regard. Recently, Johnston and colleagues (21) also reported an abnormally increased hippocampal activity during loss events, which has been associated with self-reported depression and hopelessness in a sample of 20 patients with treatment-resistant MDD and 21 healthy controls. According to the assumptions of Deakin (22), the authors hypothesized that the failure to deactivate the hippocampus during loss events is mediated by the abnormal median raphe nucleus functioning, which is normally implicated in mediating resilience and is usually able to inhibit rehearsal of aversive memories. This is also confirmed by the "dorsal raphe nucleus-periaqueductal gray-amygdala-striatum" hypothesis about depressive illness, which has been initially postulated by Deakin and Graeff (23). This theory suggested that major 


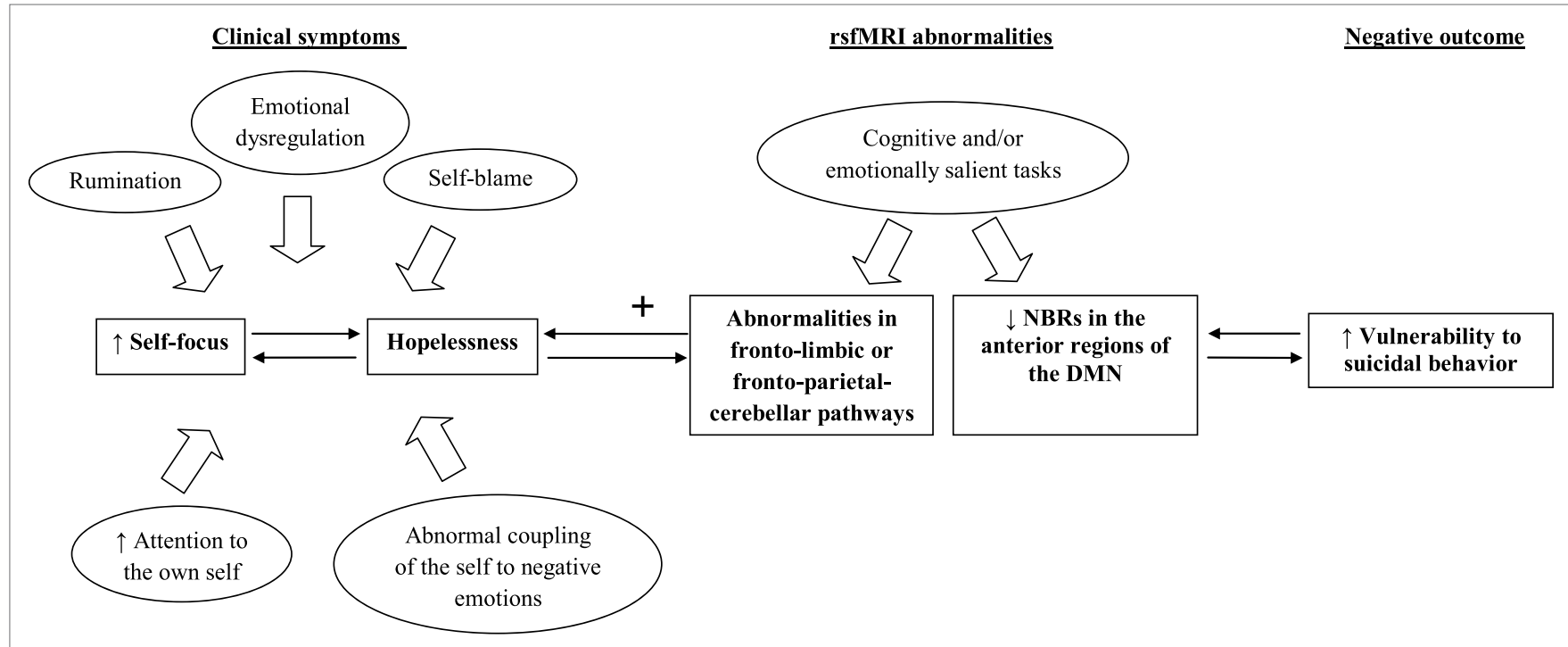

FIGURE 1 | The relationship between fMRI abnormalities and vulnerability to suicidal behavior. Note: default mode network (DMN), negative blood oxygenation level-dependent responses (NBRs), resting-state functional magnetic resonance imaging (rsfMRI).

depression is a complex condition including anxiety symptoms associated with the overactivity of dorsal raphe nucleus and its projections to the amygdala, helplessness related to the overactivity of periaqueductal gray, anhedonia associated with the overactivity of caudate/striatum as well as ruminations related to the underactivity of median raphe nucleus and its projections to the hippocampus (23).

Finally, there are also imaging evidence that investigated suicidality as a secondary emergence of disturbed experience of self in subjects with personality disorders. For example, borderline personality disorders (BPD) may be significantly associated with suicidal behavior as subjects with this psychiatric condition frequently exhibit recurrent suicidal threats, gestures, behavior, or self-mutilation $(9,24)$. In particular, Oumaya and colleagues (24) reported that self-mutilating suicide attempters may be more likely to experience feelings of depression and hopelessness, impulsivity, and affective instability, but they may also underestimate the lethality of suicidal behavior. $\mathrm{Xu}$ and colleagues (25), in the effort to identify an objective test to assist the clinical diagnosis of BPD, analyzed a sample of 21 patients with BPD and 10 healthy controls and reported that the most discriminating deficits between the two groups were located in the left medial orbitofrontal cortex, the left thalamus, and the right rostral anterior cingulate cortex. Other researchers (26) used rsfMRI to investigate changes in functional connectivity in a sample of 32 subjects with antisocial personality disorder (APD) and 35 healthy controls. Both functional and structural deficits of the precuneus, the superior parietal gyrus, and the cerebellum that may underlie the low arousal, high impulsivity, lack of conscience, cold-bloodedness, and decision-making deficits have been reported in subjects with APD when compared to healthy controls exposing them to a greater risk for suicide.

\section{MAIN SHORTCOMINGS AND FUTURE PERSPECTIVES}

Overall, rsfMRI studies suggest the potential to identify functional intermediate phenotypes that may provide interesting information about suicide risk identification.

However, rsfMRI studies need to be considered in the light of several limitations. First, based on the current knowledge about this topic, it is unclear whether the reported abnormalities represent risk markers for suicide or are directly related to the course of illness as a result of disease processes. Unfortunately, a comprehensive understanding of the altered mechanisms involved at the level of dysfunctional brain networks in subjects at risk for suicide is still lacking.

In addition, rsfMRI studies are performed using inferences at the group level according to traditional statistical hypotheses and the predictive potential of the patterns may be unlikely generalized when applying to new individuals (25). Moreover, existing rsfMRI studies usually include relatively small and clinically heterogeneous samples (e.g., predominantly suicidal young adults) that may have seriously limited their statistical power. Furthermore, subject motion during the scan may significantly influence rsfMRI findings, although several methods have been proposed including approaches to reduce the impact of motion artifacts. The potential effect of psychoactive medications in rsfMRI studies cannot be ruled out as well. Almost all of the patients who have been recruited by the existing studies were on psychotropic medications due to ethical considerations. Studies may also lack detailed information regarding medication doses or the duration of treatment.

In conclusion, elucidating the functional deficits associated with specific network disturbances may help to clarify the pathophysiological mechanisms underlying suicidal 
behavior and assist in identifying high-risk individuals in clinical practice. However, further studies involving larger samples of non-medicated individuals are needed to investigate the nature of the relationship between rsfMRI data and suicidal behavior. These additional researches could focus on examining at-risk individuals and relatives of affected subjects or shifts in brain networks before and after the emergence of suicidal ideation/ attempts in order to clarify specific causal pathways related to the observed network abnormalities. The utilization of multivariate algorithms to obtain information from multiple domains, such as neural networks, genetic and epigenetic markers, self-report

\section{REFERENCES}

1. Mackey AB, Tamas RL, Tasman A. The predicament of prediction. In: Pompili M, editor. Suicide from a Global Perspective: Risk Assessment and Management. New York: Nova Science Publishers, Inc. (2012). p. 37-42.

2. Buxton RB. The physics of functional magnetic resonance imaging (fMRI). Rep Prog Phys (2013) 76:096601. doi:10.1088/0034-4885/76/9/096601

3. Jollant F, Lawrence NS, Giampietro V, Brammer MJ, Fullana MA, Drapier $\mathrm{D}$, et al. Orbitofrontal cortex response to angry faces in men with histories of suicide attempts. Am J Psychiatry (2008) 165:740-8. doi:10.1176/appi. ajp.2008.07081239

4. Matthews S, Spadoni A, Knox K, Strigo I, Simmons A. Combat-exposed war veterans at risk for suicide show hyperactivation of prefrontal cortex and anterior cingulate during error processing. Psychosom Med (2012) 74:471-5. doi:10.1097/PSY.0b013e31824f888f

5. Jollant F, Lawrence NS, Olie E, O’Daly O, Malafosse A, Courtet P, et al. Decreased activation of lateral orbitofrontal cortex during risky choices under uncertainty is associated with disadvantageous decision-making and suicidal behavior. Neuroimage (2010) 51:1275-81. doi:10.1016/j. neuroimage.2010.03.027

6. Carter AR, Shulman GL, Corbetta M. Why use a connectivity-based approach to study stroke and recovery of function? Neuroimage (2012) 62:2271-80. doi:10.1016/j.neuroimage.2012.02.070

7. Buckner RL, Andrews-Hanna JR, Schacter DL. The brain's default network: anatomy, function, and relevance to disease. Ann N Y Acad Sci (2008) 1124:1-38. doi:10.1196/annals.1440.011

8. Coutinho JF, Fernandesl SV, Soares JM, Maia L, Gonçalves ÓF, Sampaio A. Default mode network dissociation in depressive and anxiety states. Brain Imaging Behav (2016) 10(1):147-57. doi:10.1007/s11682-015-9375-7

9. Desmyter S, van Heeringen C, Audenaert K. Structural and functional neuroimaging studies of the suicidal brain. Prog Neuropsychopharmacol Biol Psychiatry (2011) 35:796-808. doi:10.1016/j.pnpbp.2010.12.026

10. Cao J, Chen JM, Kuang L, Ai M, Fang WD, Gan Y, et al. Abnormal regional homogeneity in young adult suicide attempters with no diagnosable psychiatric disorder: a resting state functional magnetic imaging study. Psychiatry Res (2015) 231:95-102. doi:10.1016/j.pscychresns.2014.10.011

11. Northoff G. Spatiotemporal psychopathology I: no rest for the brain's resting state activity in depression? J Affect Disord (2016) 190:854-66. doi:10.1016/j. jad.2015.05.007

12. Reisch T, Seifritz E, Esposito F, Wiest R, Valach L, Michel K. An fMRI study on mental pain and suicidal behavior. J Affect Disord (2010) 126:321-5. doi:10.1016/j.jad.2010.03.005

13. Pompili M, Serafini G, Innamorati M, Biondi M, Siracusano A, Di Giannantonio $\mathrm{M}$, et al. Substance abuse and suicide risk among adolescents. Eur Arch Psychiatry Clin Neurosci (2012) 262:469-85. doi:10.1007/s00406-012-0292-0

14. Pompili M, Innamorati M, Gonda X, Serafini G, Sarno S, Erbuto D, et al. Affective temperaments and hopelessness as predictors of health and social functioning in mood disorder patients: a prospective follow-up study. J Affect Disord (2013) 150:216-22. doi:10.1016/j.jad.2013.03.026

15. Beck AT, Brown G, Berchick RJ, Stewart BL, Steer RA. Relationship between hopelessness and ultimate suicide: a replication with psychiatric outpatients. Am J Psychiatry (1990) 147:190-5. doi:10.1176/ajp.147.2.190 instruments, clinical interviews, and support diagnosis and treatment selection, are recommended as well.

\section{AUTHOR CONTRIBUTIONS}

GS conceived, designed, and drafted the present manuscript. MPa participated in the concept and helps in reviewing the current literature about the main topic. MPo critically reviewed the paper. MA and PG provided the intellectual impetuous and supervised the writing of the manuscript. All authors approved the final version of the manuscript.

16. Northoff G, Heinzel A, de Greck M, Bermpohl F, Dobrowolny H, Panksepp J. Self-referential processing in our brain - a meta-analysis of imaging studies on the self. Neuroimage (2006) 31:440-57. doi:10.1016/j. neuroimage.2005.12.002

17. Grimm S, Ernst J, Boesiger P, Schuepbach D, Boeker H, Northoff G. Reduced negative BOLD responses in the default-mode network and increased self-focus in depression. World J Biol Psychiatry (2011) 12:627-37. doi:10.3109/156 22975.2010.545145

18. Grunebaum MF, Keilp J, Li S, Ellis SP, Burke AK, Oquendo MA, et al. Symptom components of standard depression scales and past suicidal behavior. J Affect Disord (2005) 87:73-82. doi:10.1016/j.jad.2005.03.002

19. Northoff G. Psychopathology and pathophysiology of the self in depression - neuropsychiatric hypothesis. JAffect Disord (2007) 104:1-14. doi:10.1016/j.jad.2007.02.012

20. Lener MS, Iosifescu DV. In pursuit of neuroimaging biomarkers to guide treatment selection in major depressive disorder: a review of the literature. Ann N Y Acad Sci (2015) 1344:50-65. doi:10.1111/nyas.12759

21. Johnston BA, Tolomeo S, Gradin V, Christmas D, Matthews K, Steele JD. Failure of hippocampal deactivation during loss events in treatment-resistant depression. Brain (2015) 138(Pt 9):2766-76. doi:10.1093/brain/ awv177

22. Deakin J. The origins of ' 5 -HT and mechanisms of defence' by Deakin and Graeff: a personal perspective. J Psychopharmacol (2013) 27:1084-9. doi:10.1177/0269881113503508

23. Deakin JF, Graeff FG. 5-HT and the mechanisms of defence. J Psychopharmacol (1991) 5:305-15. doi:10.1177/026988119100500414

24. Oumaya M, Friedman S, Pham A, Abou Abdallah T, Guelfi JD, Rouillon F Borderline personality disorder, self-mutilation and suicide: literature review. Encephale (2008) 34:452-8. doi:10.1016/j.encep.2007.10.007

25. Xu T, Cullen KR, Houri A, Lim KO, Schulz SC, Parhi KK. Classification of borderline personality disorder based on spectral power of resting-state fMRI. Conf Proc IEEE Eng Med Biol Soc (2014) 2014:5036-9. doi:10.1109/ EMBC.2014.6944756

26. Tang Y, Jiang W, Liao J, Wang W, Luo A. Identifying individuals with antisocial personality disorder using resting-state fMRI. PLoS One (2013) 8:e60652. doi:10.1371/journal.pone.0060652

Conflict of Interest Statement: The authors declare that the research was conducted in the absence of any commercial or financial relationships that could be construed as a potential conflict of interest.

The reviewer JW and handling Editor declared their shared affiliation, and the handling Editor states that the process nevertheless met the standards of a fair and objective review.

Copyright (c) 2016 Serafini, Pardini, Pompili, Girardi and Amore. This is an open-access article distributed under the terms of the Creative Commons Attribution License (CC BY). The use, distribution or reproduction in other forums is permitted, provided the original author(s) or licensor are credited and that the original publication in this journal is cited, in accordance with accepted academic practice. No use, distribution or reproduction is permitted which does not comply with these terms. 